JOURNAL OF

SYMPLECTIC GEOMETRY

Volume 8, Number 1, 37-55, 2010

\title{
WEAK MIRROR SYMMETRY OF LIE ALGEBRAS
}

\author{
Richard Cleyton, Jorge Lauret, and Yat Sun Poon
}

Weak mirror symmetry relates a manifold with complex structure to another manifold equipped with a symplectic structure through a quasiisomorphism of associated differential Gerstenhaber algebras. The two manifolds are then mirror partners. In this paper we consider the analogous problem on Lie algebras. In particular we show that the semi-direct product of a Lie algebra equipped with a torsion-free flat connection with itself is a mirror partner of a semi-direct product of the same Lie algebra with its dual space. For nilpotent algebras this analysis on Lie algebras can be applied to the compact quotients of the underlying nilpotent group. We classify mirror pairs among 6-dimensional nilpotent Lie algebras that have the semi-direct product structure as well as mirror pairs admitting the more involved special Lagrangian structure, namely compatible complex and symplectic structures on the same space.

\section{Introduction}

It is well known that deformation theory of geometric objects such as complex structures and symplectic structures are dictated by a differential Gerstenhaber algebra (a.k.a. DGA) and the associated cohomology theory [23, 30]. Therefore, DGA plays a key role in mirror symmetry. In developing the algebraic aspects of mirror symmetry, Merkulov proposes the concept of weak mirror symmetry [25]. If $M$ is a manifold with a complex structure $J$ and $M^{\vee}$ is another manifold with a symplectic structure $\omega$, then $(M, J)$ and $\left(M^{\vee}, \omega\right)$ form a weak mirror pair if the associated differential Gerstenhaber algebras $\operatorname{DGA}(M, J)$ and $\operatorname{DGA}\left(M^{\vee}, \omega\right)$ are quasi-isomorphic. The goal of this paper is to construct mirror pairs when the manifolds $M$ and $M^{\vee}$ are nilmanifolds, $J$ is an invariant complex structure and $\omega$ is an invariant symplectic structure. 
In the SYZ-conjecture, one considers the geometry of special Lagrangian fibrations in a Calabi-Yau manifold $L \hookrightarrow M \rightarrow B$ with $L$ being a real threedimensional torus. The mirror image is a Calabi-Yau manifold $M^{\vee}$ with the special Lagrangian fibrations $L^{*} \hookrightarrow M^{\vee} \rightarrow B$ where $L^{*}$ is the dual torus of $L[\mathbf{2 9}]$. One way to adapt the structure of a Lie group $H$ to resemble this situation is by insisting that the Lie algebra $\mathfrak{h}$ is a semi-direct sum of a subalgebra $\mathfrak{g}$ with an abelian ideal $V$. The group $H$ is then a semi-direct product, namely the product of the group $G$ corresponding to $\mathfrak{g}$ with $V$. By restricting our attention to invariant structures on homogenous spaces of such groups, the geometry of the fibration of $H$ over $G$ is encoded in the corresponding objects on the Lie algebra $\mathfrak{h}$ of $H$. We shall speak somewhat sloppily of $\mathfrak{g}$ as the base and of $V$ as the fiber of the fibration.

Forgetting about the SYZ-conjecture, semi-direct products are still natural objects to study in connection with "weak mirror symmetry." This is so since the direct sum of the bundle of $(1,0)$-vectors $T^{(1,0)}$ and bundle of $(0,1)$ forms $T^{*(0,1)}$ on a complex manifold carries a natural Lie bracket (Schouten) such that $T^{(1,0)}$ is a sub-algebra and $T^{*(0,1)}$ is an abelian ideal. It is the associated exterior algebra of this semi-direct sum and the associated $\bar{\partial}$-complex that eventually control the deformations of the complex structure.

It is well known that a symplectic structure defines a torsion-free flat connection on a Lie algebra $\mathfrak{g}[\mathbf{1 3}]$. As we shall see, a torsion-free flat connection on a Lie algebra $\mathfrak{g}$ also defines a symplectic form $\omega$, not on $\mathfrak{g}$ but on a semidirect product $\mathfrak{h}^{\vee}$ of $\mathfrak{g}$ with its dual $\mathfrak{g}^{*}[\mathbf{1 0}]$. This symplectic form is defined such that it is Lagrangian with respect to both the base $\mathfrak{g}$ and the fiber $V^{*}=\mathfrak{g}^{*}$ and so we call the pair $\left(\mathfrak{h}^{\vee}, \omega\right)$ a Lagrangian semi-direct product. A torsion-free flat connection on $\mathfrak{g}$ also defines a totally real semi-direct product $(\mathfrak{h}, J)$ where $J$ is a complex structure on a semi-direct sum of $\mathfrak{g}$ with itself. Such complex structures are particular cases of complex product structures [1]. The observation that both complex structures and symplectic structures on certain semi-direct products are related to the notion torsion-free flat connections may be found in [5]. Torsion-free flat connections are also known as affine structures and as such already have widespread application in the study of mirror symmetry, see for instance $[\mathbf{2}, \mathbf{8}, \mathbf{1 9}]$. Left-invariant torsion-free flat connections on Lie groups are equivalent to (Lie compatible) left-symmetric algebras. Much is known about left-symmetric algebras. In particular existence problems have been examined and it is known that no left-symmetric structure exists on semi-simple algebras. Also, certain nilpotent algebras of dimension greater than nine have no left-symmetric structures $[\mathbf{6}, \mathbf{9}, \mathbf{1 1}, \mathbf{1 2}]$.

Therefore we confine the scope of our present paper to deal with solvable spaces. Recent advance in resolving the Benson-Gordon conjecture means that when we insist that $\mathfrak{h}$ should carry a Kähler structure then $\mathfrak{h}$ is flat and therefore of a very special solvable type $[\mathbf{4}, \mathbf{7}, \mathbf{2 1}]$. For a nilpotent 
algebra more is true - it is Kähler only if it is abelian $[\mathbf{3}, \mathbf{2 0}]$. Therefore an invariant symplectic form $\omega$ on a non-abelian nilpotent algebra is of type $(1,1)$ with respect to a complex structure $J$ if and only if $\omega$ and $J$ determine a non-definite metric. We call such a pair a pseudo-Kählerian geometry.

Invariant complex structures, their Dolbeault cohomology and pseudoKählerian geometry on nilmanifolds have been a subject of much investigation in recent years, especially when the complex dimension is equal to three $[\mathbf{1 5 - 1 7}, \mathbf{2 7}, \mathbf{2 8}]$. In particular, with the recent advance in understanding the cohomology theory on nilmanifolds [27], our computation and results on nilpotent algebras in this paper could be used to provide a full description of the differential Gerstenhaber algebras of any invariant complex structures on nilmanifolds in all dimensions.

This paper is organized as follows. In the next section, we briefly review the construction of differential Gerstenhaber algebras for complex and symplectic structures, the definition of semi-direct products and establish notations for subsequent computation. In Section 3, we study the complex and symplectic geometry on semi-direct products. The key observation in this section is Theorem 3.10, which states that the differential Gerstenhaber algebra on a totally real semi-direct product is isomorphic to the differential Gerstenhaber algebra of the Lagrangian dual semi-direct product as constructed in Proposition 3.2.

In Section 4, we focus on nilpotent algebras of dimension-six. Note that the two-dimensional case is trivial, and the four-dimensional case is already tackled in [26]. Our first step in addressing the issue of finding mirror pairs of special Lagrangian nilpotent algebras in dimension-six begins in the proof of Theorem 4.1, which identifies the weak mirror image of a totally real semidirect product. After an analysis on the existence of special Lagrangian semidirect products, the main result is Theorem 4.3, which provides a complete list of weak mirror pairs of special Lagrangian nilpotent algebras in sixdimension.

\section{Preliminaries}

We first recall two well-known constructions of DGA $[\mathbf{1 4}, \mathbf{2 5}, \mathbf{3 0}]$. After a motivation due to weak mirror symmetry, we recall the definition of semidirect product of Lie algebras.

2.1. DGA of a complex structure. Suppose $J$ is an integrable complex structure on $\mathfrak{h}$. i.e., $J$ is an endomorphism of $\mathfrak{h}$ such that $J \circ J=-1$ and

$$
[x \bullet y]+J[J x \bullet y]+J[x \bullet J y]-[J x \bullet J y]=0 .
$$

Then the $\pm i$ eigenspaces $\mathfrak{h}^{(1,0)}$ and $\mathfrak{h}^{(0,1)}$ are complex Lie subalgebras of the complexified algebra $\mathfrak{h}_{\mathbb{C}}$. Let $\mathfrak{f}$ be the exterior algebra generated by 
$\mathfrak{h}^{(1,0)} \oplus \mathfrak{h}^{*(0,1)}$, i.e.,

$$
\mathfrak{f}^{n}:=\wedge^{n}\left(\mathfrak{h}^{(1,0)} \oplus \mathfrak{h}^{*(0,1)}\right) \quad \text { and } \quad \mathfrak{f}=\oplus_{n} \mathfrak{f}^{n} .
$$

The integrability condition in (2.1) implies that $\mathfrak{f}^{1}$ is closed under the Schouten bracket

$$
[x+\alpha \bullet y+\beta]:=[x, y]+\iota_{x} \mathrm{~d} \beta-\iota_{y} \mathrm{~d} \alpha .
$$

Let $d$ be the Chevalley-Eilenberg (C-E) differential $d$ for the Lie algebra $(\mathfrak{h},[-\bullet-])$. Then $\left(\wedge \mathfrak{h}^{*}, d\right)$ is a differential graded algebra. Similarly, let $\bar{\partial}$ be the C-E differential for the complex Lie algebra $\overline{\mathfrak{f}}^{1}$. As the natural pairing on $\left(\mathfrak{h} \oplus \mathfrak{h}^{*}\right) \otimes \mathbb{C}$ induces a complex linear isomorphism $\left(\overline{\mathfrak{f}}^{1}\right)^{*} \cong \mathfrak{f}^{1}$, the C-E differential of the Lie algebra $\overline{\mathfrak{f}}^{1}$ is a map from $\mathfrak{f}^{1}$ to $\mathfrak{f}^{2}$. It turns out that $(\mathfrak{f},[-\bullet-], \wedge, \bar{\partial})$ form a differential Gerstenhaber algebra which we denote by $\operatorname{DGA}(\mathfrak{h}, J)$. The same construction shows that $(\overline{\mathfrak{f}},[-\bullet-], \wedge, \partial)$ is a DGA, conjugate linearly isomorphic to $\operatorname{DGA}(\mathfrak{h}, J)$. The above construction could be carried out similarly on a manifold with a complex structure.

2.2. DGA of a symplectic structure. Let $\mathfrak{k}$ be a Lie algebra over $\mathbb{R}$. Suppose that $\omega$ is a symplectic form on $\mathfrak{k}$. Then the contraction with $\omega$, $\omega: \mathfrak{k} \rightarrow \mathfrak{k}^{*}$ is a real non-degenerate linear map. Define a bracket on $\mathfrak{k}^{*}$ by

$$
[\alpha \bullet \beta]_{\omega}:=\omega\left[\omega^{-1} \alpha \bullet \omega^{-1} \beta\right] .
$$

It is a tautology that $\left(\mathfrak{k}^{*},[-\bullet-]_{\omega}\right)$ becomes a Lie algebra, with the map $\omega$ understood as a Lie algebra homomorphism.

In addition, the exterior algebra of the dual $\mathfrak{h}^{*}$ with the $\mathrm{C}-\mathrm{E}$ differential $d$ for the Lie algebra $\mathfrak{k}$ is a differential graded Lie algebra. In fact, $\left(\wedge^{\bullet} \mathfrak{k}^{*},[-\bullet-]_{\omega}, \wedge, d\right)$ is a DGA over $\mathbb{R}$. After complexification we denote this by $\operatorname{DGA}(\mathfrak{k}, \omega)$.

\subsection{Quasi-isomorphisms and isomorphisms.}

Definition 2.1 [25]. The Lie algebra $\mathfrak{h}$ with an integrable complex structure $J$ and the Lie algebra $\mathfrak{k}$ with a symplectic structure $\omega$ form a weak mirror pair if the DGAs DGA $(\mathfrak{h}, J)$ and DGA $(\mathfrak{k}, \omega)$ are quasi-isomorphic.

Suppose that $\phi: \operatorname{DGA}(\mathfrak{k}, \omega) \rightarrow \operatorname{DGA}(\mathfrak{h}, J)$ is a quasi-isomorphism. Since the concerned DGAs are exterior algebras generated by finite-dimensional Lie algebras, it is natural to examine the property of the Lie algebra homomorphism on the spaces of degree-one elements.

$$
\phi: \mathfrak{k}_{\mathbb{C}}^{*} \rightarrow \mathfrak{f}^{1}=\mathfrak{h}^{(1,0)} \oplus \mathfrak{h}^{*(0,1)} .
$$

In particular, if the restriction of $\phi$ to $\mathfrak{k}_{\mathbb{C}}^{*}$ is an isomorphism, it induces an isomorphism from $\operatorname{DGA}(\mathfrak{h}, J)$ to $\operatorname{DGA}(\mathfrak{k}, \omega)$. It turns out that for a special class of algebras, this is the only situation when quasi-isomorphism occurs. 
Proposition 2.2 [14]. Suppose that $\mathfrak{h}$ and $\mathfrak{k}$ are finite-dimensional nilpotent Lie algebras of the same dimension, $J$ is an integrable complex structure on $\mathfrak{h}$ and $\omega$ is a symplectic form on $\mathfrak{k}$. Then a homomorphism $\phi$ from $\operatorname{DGA}(\mathfrak{h}, J)$ to $\mathrm{DGA}(\mathfrak{k}, \omega)$ is a quasi-isomorphism if and only if it is an isomorphism.

This proposition provides a large class of Lie algebras to work on. So in this paper we focus our attention on a restricted type of weak mirror pairs. Namely, we seek a pair such that the map $\phi$ in (2.5) is an isomorphism on the degree-one level. Since the Lie algebra $\mathfrak{k}^{*}$ is tautologically isomorphic to $\mathfrak{k}$ via $\omega$, we concern ourselves with the non-degeneracy of the map

$$
\phi \circ \omega: \mathfrak{k}_{\mathbb{C}} \rightarrow \mathfrak{k}_{\mathbb{C}}^{*} \rightarrow \mathfrak{f}^{1}=\mathfrak{h}^{(1,0)} \oplus \mathfrak{h}^{*(0,1)} .
$$

When this is an isomorphism one immediately obtains conditions on the structure of $\mathfrak{k}$. The reason for this is that with respect to the Schouten bracket, $\mathfrak{h}^{(1,0)}$ is a subalgebra of $\mathfrak{f}^{1}$ and $\mathfrak{h}^{*(0,1)}$ is an abelian ideal. In addition, $\operatorname{dim} \mathfrak{h}^{(1,0)}=\operatorname{dim} \mathfrak{h}^{*(0,1)}$. In other words, $\mathfrak{f}^{1}$ and $\mathfrak{k}$ are semi-direct products of a very particular form.

2.4. Semi-direct products. Let $\mathfrak{g}$ be a Lie algebra, $V$ a vector space and let $\rho: \mathfrak{g} \rightarrow \operatorname{End}(V)$ be a representation. On the vector space $\mathfrak{g} \oplus V$, define

$$
[x+u, y+v]_{\rho}:=[x, y]+\rho(x) v-\rho(y) u,
$$

where $x, y$ are in $\mathfrak{g}$ and $u, v$ are in $V$. Then this determines a Lie bracket on $\mathfrak{g} \oplus V$. This structure is a particular case of a semi-direct product. As a Lie algebra it is denoted by $\mathfrak{h}=\mathfrak{h}(\mathfrak{g}, \rho)=\mathfrak{g} \ltimes_{\rho} V$. By construction, $V$ is an abelian ideal and $\mathfrak{g}$ is a complementary subalgebra.

Conversely, suppose $V$ is an abelian ideal of a Lie algebra $\mathfrak{h}$ and $\mathfrak{g}$ a complementary subalgebra. The adjoint action of $\mathfrak{g}$ on $V$ then gives $\rho: \mathfrak{g} \rightarrow$ $\operatorname{End}(V)$.

In this paper, we are solely interested in the situation when $\operatorname{dim} \mathfrak{g}=$ $\operatorname{dim} V$. In particular, when the vector space $V$ is regarded as the underlying space of the real algebra $\mathfrak{g}$ or its dual $\mathfrak{g}^{*}$, interesting geometry and other phenomena arise through the representations of $\mathfrak{g}$ as described next.

\section{Geometry on semi-direct products}

Through left translations, there is an one-to-one correspondence between left invariant connections $\nabla$ and linear maps $\gamma: \mathfrak{g} \rightarrow \operatorname{End}(\mathfrak{g})$. At the identity of the Lie group, $\nabla_{x} y=\gamma(x) y$. The torsion $T^{\gamma}(x, y)$ of the corresponding $\nabla$ is $[x, y]-\gamma(x) y+\gamma(y) x$, and its curvature $R^{\gamma}$ is given by $\gamma([x, y])-$ $\gamma(x) \gamma(y)+\gamma(y) \gamma(x)$. Since all connections considered here are left-invariant, linear maps $\gamma: \mathfrak{g} \rightarrow \operatorname{End}(\mathfrak{g})$ are referred to as connections on $\mathfrak{g}$ and say that $\gamma$ is flat if $R^{\gamma}=0$ and torsion-free if $T^{\gamma}=0$. 
3.1. Totally real semi-direct products. A complex structure on a real vector space $W$ is an endomorphism $J$ such that $J^{2}=-1$. If $V$ is a subspace of $W$ such that $V \oplus J V=W$, we say that $J$ is totally real with respect to $V$. Given a totally real $J$ any $w$ in $W$ may be written uniquely as $w=x+J y$ for $x, y$ in $V$. So $W \cong V \oplus V$ and $J$ may be viewed as the standard complex structure $J_{0}$ on $V \oplus V$ given by $(x, y) \mapsto(-y, x)$.

If $\mathfrak{g}=(W,[\cdot, \cdot])$ is a Lie algebra we say that $J$ is integrable if the Nijenhuis tensor

$$
N_{J}(x, y):=[x, y]-[J x, J y]+J([x, J y]+[J x, y])
$$

is zero for all $x$ and $y$ in $W$. If $J$ is totally real with respect to $V$ then $J$ is integrable if and only if $N_{J}(x, y)=0$ for all $x, y \in V$. This follows by the identity $N_{J}(x, y)=J N_{J}(x, J y)$ valid for all $x, y \in W$.

Definition 3.1. Suppose that $\mathfrak{h}(\mathfrak{g}, \rho)=\mathfrak{g} \ltimes_{\rho} V$ is a semi-direct product Lie algebra. A complex structure on $\mathfrak{g} \ltimes_{\rho} V$ is totally real if $J \mathfrak{g}=V$.

Since $V$ is an abelian ideal, $[J x, J y]=0$ for all $x, y$ in $\mathfrak{g}$ and so the Nijenhuis tensor vanishes precisely when

$$
[x, y]+J \rho(x) J y-J \rho(y) J x=0 .
$$

for all $x, y \in \mathfrak{g}$. This has the significance that

$$
\gamma(x) y:=-J \rho(x) J y
$$

defines a torsion-free flat connection on $\mathfrak{g}$.

On the other hand, take a torsion-free flat connection $\gamma$ on $\mathfrak{g}$. The totally real complex structure $J$ on $\mathfrak{h}:=\mathfrak{g} \ltimes_{\gamma} \mathfrak{g}$ defined by $J(x, y)=(-y, x)$ becomes integrable with respect to $[\cdot, \cdot]_{\gamma}$. In summary, we have an observation which is at least implicitly contained in $[\mathbf{1}]$.

Proposition 3.2. There is a one-to-one correspondence between torsionfree flat connections on $\mathfrak{g}$ and totally real integrable complex structures on semi-direct products $\mathfrak{g} \ltimes_{\rho} V$.

3.2. Lagrangian semi-direct products. Let $\omega$ be a two-form on an evendimensional vector space $W$, i.e., $\omega \in \Lambda^{2} W^{*}$. As a linear map $\omega: W \rightarrow W^{*}$, $\omega^{*}=-\omega$. Then $\omega$ is non-degenerate if $\omega: W \rightarrow W^{*}$ is invertible.

A subspace $V$ of $W$ is Lagrangian if $\omega(V)=\operatorname{Ann}(V)$. A splitting $W=$ $V \oplus V^{\prime}$ of a vector space $W$ into a direct sum is called Lagrangian with respect to $\omega$ if both $V$ and $V^{\prime}$ are Lagrangian with respect to $\omega$.

Let $V$ be a vector space. Then $V \oplus V^{*}$ carries a two-form $\omega$ given by the canonical pairing $\omega(x, \alpha)=\alpha(x)$ for $\alpha \in V^{*}$ and $x \in V$. The given splitting is Lagrangian. It serves as the standard model for any Lagrangian splitting. Explicitly, if $W=V \oplus V^{\prime}$ and $x \in V, x^{\prime} \in V^{\prime}$, then the map $f\left(x+x^{\prime}\right):=x+\omega\left(x^{\prime}\right)$ is an isomorphism from $W$ to $V \oplus V^{*}$, intertwining the non-degenerate 2 -forms. 
Suppose that $\mathfrak{g}=(W,[\cdot, \cdot])$ is a Lie algebra. Then the derivative of a twoform $\omega$ with respect to the $\mathrm{C}-\mathrm{E}$ differential of $\omega$ is closed if and only if for all $x, y$,

$$
\omega([x, y])=\operatorname{ad}^{*}(x)(\omega(y))-\operatorname{ad}^{*}(y)(\omega(x))
$$

where $\left(\operatorname{ad}^{*}(x) \alpha\right)(y)=-\alpha([x, y])$. If $\omega$ is also non-degenerate, $\omega$ is a symplectic form.

Definition 3.3. Suppose that $\mathfrak{h}=\mathfrak{g} \ltimes_{\rho} V$ is a semi-direct product. It is said to be Lagrangian with respect to a symplectic form $\omega$ if the subalgebra $\mathfrak{g}$ and the abelian ideal $V$ are both Lagrangian.

When $\mathfrak{g} \ltimes_{\rho} V$ is Lagrangian, then $\omega(V) \cong \mathfrak{g}^{*}$, and $\omega(\mathfrak{g}) \cong V^{*}$. Define

$$
\rho^{*}: \mathfrak{g} \rightarrow \operatorname{End}\left(V^{*}\right) \quad \text { by } \quad\left(\rho^{*}(x) \alpha\right)(u)=-\alpha(\rho(x) u) .
$$

Then $\left(\rho^{*}(x) \omega(y)\right)(u)=-\omega(y, \rho(x) u)=\left(\operatorname{ad}_{\rho}^{*}(x) \omega(y)\right)(u)$. Comparing to equation (3.10) it is now clear that $\omega$ is closed if and only if

$$
\omega([x, y])=\rho^{*}(x)(\omega(y))-\rho^{*}(y)(\omega(x))
$$

for all $x, y \in \mathfrak{g}$. It follows that $\gamma(x) y:=\omega^{-1}\left(\rho^{*}(x) \omega(y)\right)$ defines a torsion-free flat connection on $\mathfrak{g}$.

Conversely, take a torsion-free flat connection $\gamma$ on $\mathfrak{g}$. Let $\omega$ be the standard skew pairing on $\mathfrak{g} \oplus \mathfrak{g}^{*}: \omega(x+u, y+v)=u(y)-v(x)$. Define the bracket on $\mathfrak{g} \oplus \mathfrak{g}^{*}$ as the semi-direct product by representation $\gamma^{*}$. Then $\mathrm{d} \omega=0$ and the semi-direct product is Lagrangian with respect to $\omega$. This gives us the following result.

Proposition 3.4 [10]. There is a one-to-one correspondence between torsion-free flat connections $\rho$ on Lie algebras $\mathfrak{g}$ and Lagrangian semi-direct products $\mathfrak{g} \ltimes_{\rho} V$.

3.3. From complex structure to two-form, and back. Propositions 3.2 and 3.4 yield a one-to-one correspondence between certain integrable complex structures and certain symplectic forms via torsion-free flat connections. It is now easy to construct a direct relation between two-form and complex structures.

Suppose $W=V \oplus J V$. On $W^{\vee}:=V \oplus(J V)^{*}$, define

$$
\omega_{J}(x+u, y+v):=v(J x)-u(J y),
$$

where $x, y$ are in $V$ and $u, v$ are in $(J V)^{*}$. Then $\omega_{J}$ is non-degenerate on $W^{\vee}$ with both $V$ and $(J V)^{*}$ being Lagrangian.

Conversely suppose $\omega$ is a non-degenerate 2-form on $W=V \oplus V^{\prime}$ with both $V$ and $V^{\prime}$ being Lagrangian. Write $V^{\prime}=\omega^{-1}\left(V^{*}\right)$ and set

$$
J_{\omega}(x+u)=-\omega^{-1}(u)+\omega(x)
$$

for all $x+u$ in $V \oplus V^{*}$. Clearly, both $V$ and $V^{*}$ are totally real with respect to $J_{\omega}$. Therefore, we have the following. 
Lemma 3.5. There is a one-to-one correspondence between totally real integrable complex structure $J$ on a semi-direct product $\mathfrak{h}=\mathfrak{g} \ltimes_{\rho} V$ and symplectic structure $J_{\omega}$ on the semi-direct product $\mathfrak{h}^{\vee}=\mathfrak{g} \ltimes_{\rho^{*}} V^{*}$. The correspondence is given by (3.13) and (3.14).

3.4. Special Lagrangian structures. A non-degenerate two-form $\omega$ and a complex structure $J$ on a vector space $W$ are said to be compatible if $\omega(J \xi, J \eta)=\omega(\xi, \eta)$ for all $\xi, \eta$ in $W$. In such case $g(\xi, \eta):=\omega(\xi, J \eta)$ is a non-degenerate symmetric bilinear form on $W$, the induced metric for which $J$ is an orthogonal transformation.

Suppose that $\omega$ and $J$ are compatible and a vector subspace $V$ is totally real with respect $J$. Then $V$ is isotropic with respect to $\omega$ if and only if $J V$ is isotropic, and the splitting $W=V \oplus J V$ is orthogonal with respect to the induced metric. Conversely, let $J$ be a complex structure on $W$ and $V$ a totally real subspace. Any inner product $g$ on $V$ could be extended to $W$ by declaring $g(J x, J y)=g(x, y)$ for $x, y \in V$ and $g(x, J y)=0$. Then $\omega$ and $J$ are compatible.

Suppose that $W$ is a Lie algebra $\mathfrak{h}=(W,[\cdot, \cdot])$, and $\omega$ is a symplectic form and $J$ is an integrable complex structure. If $\omega$ and $J$ are compatible, the pair is called a pseudo-Kähler structure.

Definition 3.6. Let $\mathfrak{h}$ be a semi-direct product $\mathfrak{h}=\mathfrak{g} \ltimes_{\rho} V$ Lie algebra. Let $(\omega, J)$ be a pseudo-Kähler structure on $\mathfrak{h}$. Then $\mathfrak{h}$ is said to be special Lagrangian if $\mathfrak{g}$ and $V$ are totally real with respect to $J$ and Lagrangian with respect to $\omega$. We then also call $(\omega, J)$ a special Lagrangian structure on the semi-direct product $\mathfrak{h}$.

Proposition 3.7. If $(\omega, J)$ is a special Lagrangian structure on a semidirect product $\mathfrak{h}=\mathfrak{g} \ltimes_{\rho} V$, then $\left(\omega_{J}, J_{\omega}\right)$ is a special Lagrangian structure on the dual semi-direct product $\mathfrak{h}^{\vee}=\mathfrak{g} \ltimes_{\rho^{*}} V$.

Proof. In view of Lemma 3.5, the only issue is to verify that for any $x+u, y+v$ in $\mathfrak{g} \oplus V^{*}, \omega_{J}\left(J_{\omega}(x+u), J_{\omega}(y+v)\right)=\omega_{J}(x+u, y+v)$. Given the compatibility of $\omega$ and $J$, the proof is simply a matter of definitions as given in (3.13) and (3.14).

Other than allowing the metric being pseudo-Kähler, Definition 3.6 above is an invariant version of the usual definition of special Lagrangian structures found in literature on mirror symmetry if we extend the metric $g$ and the complex structure $J$ to be left-invariant tensors on the simply connected Lie groups of $\mathfrak{h}$ and $\mathfrak{g}$ (see, e.g., $[\mathbf{2 2}]$ ). To illustrate this point, note that if $\left\{e^{1}, \ldots, e^{n}\right\}$ is an orthonormal basis of $\mathfrak{g}$ with respect to the pseudoRiemannian metric $g$, set $u^{j}=e^{j}+i J e^{j}$. Then $\left\{u^{1}, \ldots, u^{n}\right\}$ is a Hermitian basis of $\mathfrak{h}$. Then the Kähler form $\omega$ is

$$
\omega=i \sum_{j=1}^{n} u^{j} \wedge \bar{u}^{j}=i \sum_{j}\left(e^{j}+i J e^{j}\right) \wedge\left(e^{j}-i J e^{j}\right) .
$$


The complex volume form is

$$
\begin{aligned}
\Phi= & u^{1} \wedge \cdots \wedge u^{n}=\left(e^{1}+i J e^{1}\right) \wedge \cdots \wedge\left(e^{n}+i J e^{n}\right) \\
= & e^{1} \wedge \cdots \wedge e^{n}+i^{n} J e^{1} \wedge \cdots \wedge J e^{n} \\
& + \text { terms mixed with both } e^{j} \text { and } J e^{k} .
\end{aligned}
$$

When $n$ is odd the real part of $\Phi$ restricts to zero on $V$ and the imaginary part restricts to a real volume form. Therefore, the fibers of the quotient map from the Lie group $H$ onto $G$ are special Lagrangian submanifolds.

3.5. Flat connections and special Lagrangian structures. Suppose $(\omega, J)$ is a special Lagrangian structure on $\mathfrak{h}=\mathfrak{g} \ltimes_{\rho} V$ and let $g$ be the induced metric. Define $\gamma(x):=-J \rho(x) J$. Then $\gamma$ is a torsion-free flat connection on $\mathfrak{g}$. Let $\gamma^{\mathrm{t}}$ be the transpose of $\gamma$ with respect to $g$. i.e., $g\left(\gamma^{\mathrm{t}}(x), y\right):=g(x, \gamma(y))$. Since $\omega$ is closed and $\gamma$ is flat, $-\gamma^{\mathrm{t}}$ is also a torsion-free flat connection.

On the other hand, suppose that $\mathfrak{g}$ is equipped with a non-degenerate bilinear form $g$. Let $\gamma$ be a torsion-free flat connection such that $\gamma^{\prime}:=-\gamma^{\mathrm{t}}$ is also a torsion-free flat connection. Then, as above the complex structure on $\mathfrak{h}:=\mathfrak{g} \ltimes_{\gamma} \mathfrak{g}$ given by $J(x, y)=(-y, x)$ is integrable. We write $x+J y, x, y \in$ $\mathfrak{g}$ for the elements in $\mathfrak{h}$. Define $\omega$ on $\mathfrak{h}$ by $\omega(x, y)=\omega(J x, J y)=0$ and $\omega(x, J y)=g(x, y)=-\omega(y, J x)$ and set $g(J x, J y)=g(x, y)$. Then $\mathrm{d} \omega=0$ by virtue of $\gamma^{\prime}$ being flat and torsion-free.

Note that we may equally well choose to work with the integrable complex structure $J^{\prime}(x, y)=(-y, x)$ on $\mathfrak{h}:=\mathfrak{g} \ltimes_{\gamma^{\prime}} \mathfrak{g}$ and the associated symplectic form $\omega^{\prime}$. This is of course precisely the "mirror image" of $(\mathfrak{h}, J, \omega)$. This all amounts to the next observation.

Proposition 3.8. Let $\mathfrak{g}$ be a Lie algebra with a non-degenerate bilinear form $g$. Then there is a two-to-one correspondence between special Lagrangian structures on a semi-direct product extending the Lie algebra $\mathfrak{g}$ and torsionfree flat connections $\gamma$ on $\mathfrak{g}$ such that the dual connection $-\gamma^{\mathrm{t}}$ is also torsionfree and flat.

3.6. Canonical isomorphism of DGAs. Let $\gamma$ be a torsion-free flat connection on a Lie algebra $\mathfrak{g}$. Write $V$ for the associated representation of $\mathfrak{g}$ on itself and consider the usual integrable complex structure $J$ on $\mathfrak{h}=\mathfrak{g} \ltimes_{\gamma} V$. Then $\mathfrak{f}^{1}(\mathfrak{h}, J)=\mathfrak{h}^{(1,0)} \oplus \mathfrak{h}^{*(0,1)}$. Note that $J$ acts on $V^{*}$ by $\left(J v^{*}\right)(x+u)=$ $-v^{*}(J x+J u)=-v^{*}(J x)$. In particular, $J v^{*} \in \operatorname{Ann}(V) \subset \mathfrak{h}^{*}$.

Now set $\mathfrak{h}^{\vee}:=\mathfrak{g} \ltimes_{\gamma^{*}} V^{*}$ and define $\phi: \mathfrak{h}_{\mathbb{C}}^{\vee} \rightarrow \mathfrak{f}^{1}(\mathfrak{h}, J)$ as the tautological map:

$$
\phi\left(x+v^{*}\right):=(1-i J) x+(1-i J) v^{*} .
$$

Recall that the restriction of the Schouten bracket on the space $\mathfrak{f}^{1}(\mathfrak{h}, J)$ is a Lie bracket. It is a matter of tracing various definitions to verify the following. 
Lemma 3.9. The map $\phi: \mathfrak{h}_{\mathbb{C}}^{\vee} \rightarrow \mathfrak{f}^{1}(\mathfrak{h}, J)$ is an isomorphism of Lie algebras.

Since the complex structure $J$ on $\mathfrak{h}$ induces a symplectic structure $\omega_{J}$ on $\mathfrak{h}^{\vee}$, the contraction map $\omega_{J}: \mathfrak{h}^{\vee} \rightarrow\left(\mathfrak{h}^{\vee}\right)^{*}$ carries the Lie bracket on $\mathfrak{h}^{\vee}$ to a Lie bracket $[-\bullet-]_{\omega_{J}}$ on $\left(\mathfrak{h}^{\vee}\right)^{*}$. Therefore, $\phi \circ \omega_{J}^{-1}$ is a Lie algebra isomorphism from $\left(\mathfrak{h}^{\vee}\right)_{\mathbb{C}}^{*}$ to $\mathfrak{f}^{1}$. It induces an isomorphism from the underlying Gerstenhaber algebra of $\operatorname{DGA}\left(\mathfrak{h}^{\vee}, \omega_{J}\right)$ to that of $\operatorname{DGA}(\mathfrak{h}, J)$. Next we demonstrate that this map is also an isomorphism of differential graded algebra. i.e.,

$$
\phi \circ \omega^{-1} \circ d=\bar{\partial} \circ \phi \circ \omega^{-1}
$$

As $\phi^{*}:\left(\mathfrak{f}^{1}\right)^{*} \rightarrow\left(h_{\mathbb{C}}^{\vee}\right)^{*}$, the conjugated map is $\bar{\phi}^{*}:\left(\mathfrak{f}^{1}\right)^{*} \rightarrow\left(\overline{h_{\mathbb{C}}^{\vee}}\right)^{*}$. In the next calculation we implicitly identify the isomorphic Lie algebras $\left(\mathfrak{f}^{1}\right)^{*}$ with $\mathfrak{f}^{1}$ and $\mathfrak{h}_{\mathbb{C}}^{\vee}$ with its conjugate $\overline{\mathfrak{h}_{\mathbb{C}}^{\vee}}$. Hence $\bar{\phi}^{*}$ is identified with the map $\bar{\phi}^{*}: \mathfrak{f}^{1} \rightarrow\left(h_{\mathbb{C}}^{\vee}\right)^{*}$. Then $\bar{\phi}^{*} \phi$ is a map from $\mathfrak{h}_{\mathbb{C}}^{\vee}$ to $\left(h_{\mathbb{C}}^{\vee}\right)^{*}$. According to $[\mathbf{1 4}$, Proposition 11], the map $\phi \circ \omega_{J}^{-1}$ yields an isomorphism of differential graded algebra as in (3.15) if, up to a constant, $\bar{\phi}^{*} \phi$ is equal to the contract of $\omega_{J}$. Therefore, we have the following computation.

$$
\begin{aligned}
\left(\bar{\phi}^{*} \phi\right)\left(x+u^{*}\right)\left(y+v^{*}\right) \\
\quad=\left(\bar{\phi}^{*}\right)\left((1-i J) x+(1-i J) u^{*}\right)\left(y+v^{*}\right) \\
\quad=\overline{\left.\left((1+i J) x+(1+i J) u^{*}\right)\left((1-i J) y+(1-i J) v^{*}\right)\right)} \\
\quad=2 i\left(u^{*}(J y)-v^{*}(J x)\right)=2 i \omega_{J}\left(x+u^{*}, y+v^{*}\right) .
\end{aligned}
$$

This shows that the isomorphism $\phi$ defines a DGA structure on the de Rham complex of $\mathfrak{h}_{\mathbb{C}}^{\vee}$ isomorphic to the one defined by $\omega_{J}$, since the brackets differ only by multiplication by a constant. In particular, we have

Theorem 3.10. $\operatorname{DGA}(\mathfrak{h}, J)$ and $\operatorname{DGA}\left(\mathfrak{h}^{\vee}, \omega_{J}\right)$ are isomorphic.

A similar construction shows that for a Lagrangian symplectic form $\omega$ on $\mathfrak{h}=\mathfrak{g} \ltimes_{\rho} V$ the associated differential Gerstenhaber algebras DGA $(\mathfrak{h}, \omega)$ and $\operatorname{DGA}\left(\mathfrak{h}^{\vee}, J_{\omega}\right)$ are isomorphic.

\section{Nilpotent algebras of dimension at most six.}

In this section, we tackle two problems when the algebra $\mathfrak{h}$ is six-dimensional and nilpotent. 
Problem 1. Let $\mathfrak{h}$ be a nilpotent algebra. Suppose that it is a semi-direct product $\mathfrak{h}=\mathfrak{g} \ltimes V$ and totally real with respect to a complex structure $J$. Identify the corresponding algebra $\mathfrak{h}^{\vee}=\mathfrak{g} \ltimes V^{*}$ and the associated symplectic structure $\omega_{J}$.

The next problem raises a more restrictive issue.

Problem 2. Let $\mathfrak{h}$ be a nilpotent algebra. Suppose that it is a semi-direct product $\mathfrak{h}=\mathfrak{g} \ltimes V$ and it is special Lagrangian with respect to a pseudoKähler structure $(J, \omega)$. Identify the corresponding algebra $\mathfrak{h}^{\vee}=\mathfrak{g} \ltimes V^{*}$ and the associated pseudo-Kähler structure $\left(J^{\vee}, \omega^{\vee}\right)$.

We shall exclude the trivial algebra in subsequent computation, although we may include it for the completeness of a statement in a theorem. The first even dimension in which a non-abelian nilpotent algebra occurs is four.

There are two four-dimensional non-trivial nilpotent algebras [18]. Only one of them is a semi-direct product, namely the direct sum of a trivial algebra with a three-dimensional Heisenberg algebra. It happens to be the only one admitting integrable invariant complex structures [28, Proposition 2.3]. Up to equivalence, there exists a basis $\left\{e_{1}, e_{2}, e_{3}, e_{4}\right\}$ on the algebra $\mathfrak{h}$ such that the structure equation is simply $\left[e_{1}, e_{2}\right]=-e_{3}$. The corresponding complex structure is determined by $J\left(e_{1}\right)=e_{2}, J\left(e_{3}\right)=e_{4}$ etc. A symplectic form is $\omega=e^{1} \wedge e^{4}+e^{3} \wedge e^{2}$. Consider the subspaces $\mathfrak{g}:=\left\langle e_{2}, e_{4}\right\rangle$ and $V:=\left\langle e_{1}, e_{3}\right\rangle$. They determine a semi-direct product $\mathfrak{h}=\mathfrak{g} \ltimes_{\text {ad }} V$. It is apparent that this semi-direct product is special Lagrangian, the pair $(\omega, J)$ above. One may now work through our theory to demonstrate that the mirror image of $(\mathfrak{h}, J, \omega)$ is isomorphic to $(\mathfrak{h}, J, \omega)$ itself. More details could be found in $[\mathbf{2 6}]$.

4.1. Weak mirror pairs. In our calculation below, we assume that the algebra $\mathfrak{h}$ is six dimensional. We often express the structure equation on $\mathfrak{h}=\mathfrak{g} \ltimes_{\text {ad }} V$ in terms of the C-E differential on the dual basis $\left\{e^{1}, \ldots, e^{6}\right\}$. In particular we collect $\left(d e^{1}, \ldots, d e^{6}\right)$ in an array. We shall also adopt the shorthand notation that when $d e^{1}=e^{i} \wedge e^{j}+e^{\alpha} \wedge e^{\beta}$, then the first entry in this array is $i j+\alpha \beta[\mathbf{2 8}]$. To name six-dimensional algebras, we use the convention developed in [16].

Since the adjoint action of the nilpotent Lie algebra $\mathfrak{g}$ on the abelian ideal $V$ is a nilpotent representation, by the Engel Theorem there exists a basis $\left\{e_{2}, e_{4}, e_{6}\right\}$ of $V$ such that the matrix of any ad $x, x \in \mathfrak{g}$, is strictly lower triangular.

4.1.1. Assume that $\mathfrak{g}$ is abelian. There exists a basis $\left\{e_{1}, e_{3}, e_{5}\right\}$ of $\mathfrak{g}$ such that with respect to the ordered basis $\left\{e_{2}, e_{4}, e_{6}\right\}$ for $V$, the adjoint representation of $\mathfrak{g}$ on $V$ is given as below

$$
\rho\left(e_{1}\right)=-\left(\begin{array}{lll}
0 & 0 & 0 \\
a & 0 & 0 \\
c & b & 0
\end{array}\right), \quad \rho\left(e_{3}\right)=-\left(\begin{array}{lll}
0 & 0 & 0 \\
0 & 0 & 0 \\
d & e & 0
\end{array}\right), \quad \rho\left(e_{5}\right)=-\left(\begin{array}{lll}
0 & 0 & 0 \\
0 & 0 & 0 \\
f & 0 & 0
\end{array}\right) .
$$


Up to equivalence, we have the following

$$
\begin{gathered}
\mathfrak{h}_{3}: \quad \rho\left(e_{1}\right)=-\left(\begin{array}{lll}
0 & 0 & 0 \\
0 & 0 & 0 \\
0 & 1 & 0
\end{array}\right), \quad \rho\left(e_{3}\right)=-\left(\begin{array}{lll}
0 & 0 & 0 \\
0 & 0 & 0 \\
1 & 0 & 0
\end{array}\right), \quad \rho\left(e_{5}\right)=0 . \\
\mathfrak{h}_{8}: \quad \rho\left(e_{1}\right)=0, \quad \rho\left(e_{3}\right)=-\left(\begin{array}{lll}
0 & 0 & 0 \\
0 & 0 & 0 \\
1 & 0 & 0
\end{array}\right), \quad \rho\left(e_{5}\right)=0 . \\
\mathfrak{h}_{9}: \quad \rho\left(e_{1}\right)=-\left(\begin{array}{lll}
0 & 0 & 0 \\
1 & 0 & 0 \\
0 & 1 & 0
\end{array}\right), \quad \rho\left(e_{3}\right)=-\left(\begin{array}{lll}
0 & 0 & 0 \\
0 & 0 & 0 \\
1 & 0 & 0
\end{array}\right), \quad \rho\left(e_{5}\right)=0 .
\end{gathered}
$$

In addition, $\mathfrak{h}_{6}$ is given by $b=c=e=f=0$ and $a=d=1 . h_{17}$ is given by $a=b=1, c=0$ and $\rho\left(e_{3}\right)=\rho\left(e_{5}\right)=0$.

By taking the dual representation, we find

$$
\mathfrak{h}_{3}^{\vee}=\mathfrak{h}_{6}, \quad \mathfrak{h}_{6}^{\vee}=\mathfrak{h}_{3}, \quad \mathfrak{h}_{8}^{\vee}=\mathfrak{h}_{8}, \quad \mathfrak{h}_{9}^{\vee}=\mathfrak{h}_{9}, \quad \mathfrak{h}_{17}^{\vee}=\mathfrak{h}_{17} .
$$

However, $\mathfrak{h}_{3}$ does not admit any invariant symplectic form [18], therefore by the mirror construction, the semi-direct structure on $\mathfrak{h}_{6}$ with an abelian base is not totally real with any invariant complex structure. On the other hand, $\mathfrak{h}_{17}$ does not admit any invariant complex structure [28]. Therefore, if we assume that the semi-direct product on $\mathfrak{h}_{n}$ has an invariant complex structure, we are left with three cases: $\mathfrak{h}_{3}^{\vee}=\mathfrak{h}_{6}, \mathfrak{h}_{8}^{\vee}=\mathfrak{h}_{8}$ and $\mathfrak{h}_{9}^{\vee}=\mathfrak{h}_{9}$.

4.1.2. Assume that $\mathfrak{g}$ is non-abelian. In this case, $\mathfrak{g}$ is a threedimensional Heisenberg algebra. Thus there exists a basis $\left\{e_{1}, e_{3}, e_{5}\right\}$ of $\mathfrak{g}$ such that $\left[e_{1}, e_{3}\right]=-e_{5}$, and a basis $\left\{e_{2}, e_{4}, e_{6}\right\}$ of $V$ such that the adjoint representation of $\mathfrak{g}$ on $V$ is as follows.

$$
\rho\left(e_{1}\right)=-\left(\begin{array}{lll}
0 & 0 & 0 \\
a & 0 & 0 \\
c & b & 0
\end{array}\right), \quad \rho\left(e_{3}\right)=-\left(\begin{array}{lll}
0 & 0 & 0 \\
d & 0 & 0 \\
f & e & 0
\end{array}\right), \quad \rho\left(e_{5}\right)=-\left(\begin{array}{rrr}
0 & 0 & 0 \\
0 & 0 & 0 \\
b d-a e & 0 & 0
\end{array}\right) .
$$

If $d \neq 0$, by choosing $\left\{a e_{3}-d e_{1}, e_{3}\right\}$, we have a new set of $\left\{e_{1}, e_{3}, e_{5}\right\}$ such that $d=0$. If $a \neq 0$, we may consider the new basis $\left\{e_{1}, e_{3}-\frac{d}{a} e_{1}, e_{5}\right\}$ for $\mathfrak{g}$ and $\left\{e_{2}, a e_{4}+c e_{6}, e_{6}\right\}$ for $V$ and assume $a=1, d=0$ and $c=0$. Then

$$
\mathfrak{h}=(0,0,0,12,13, b 14-f 23+e 34+e 25) .
$$

If $e=0$, it is further reduced to $\mathfrak{h}=(0,0,0, a 12,13, b 14-f 23)$. The following becomes easy to verify.

$$
\begin{aligned}
& \mathfrak{h}_{6}: \quad \rho\left(e_{1}\right)=-\left(\begin{array}{ccc}
0 & 0 & 0 \\
1 & 0 & 0 \\
0 & 0 & 0
\end{array}\right), \quad \rho\left(e_{3}\right)=0, \quad \rho\left(e_{5}\right)=0, \\
& \mathfrak{h}_{7}: \quad \rho\left(e_{1}\right)=-\left(\begin{array}{lll}
0 & 0 & 0 \\
1 & 0 & 0 \\
0 & 0 & 0
\end{array}\right), \quad \rho\left(e_{3}\right)=-\left(\begin{array}{lll}
0 & 0 & 0 \\
0 & 0 & 0 \\
1 & 0 & 0
\end{array}\right), \quad \rho\left(e_{5}\right)=0, \\
& \mathfrak{h}_{10}: \quad \rho\left(e_{1}\right)=-\left(\begin{array}{lll}
0 & 0 & 0 \\
1 & 0 & 0 \\
0 & 1 & 0
\end{array}\right), \quad \rho\left(e_{3}\right)=0, \quad \rho\left(e_{5}\right)=0, \\
& \mathfrak{h}_{11}: \quad \rho\left(e_{1}\right)=-\left(\begin{array}{lll}
0 & 0 & 0 \\
1 & 0 & 0 \\
0 & 1 & 0
\end{array}\right) \quad \rho\left(e_{3}\right)=-\left(\begin{array}{lll}
0 & 0 & 0 \\
0 & 0 & 0 \\
1 & 0 & 0
\end{array}\right) \quad \rho\left(e_{5}\right)=0 \text {. }
\end{aligned}
$$


If $e \neq 0$, consider the new basis

$$
\left\{\ell_{1}, \ldots, \ell_{6}\right\}=\left\{e^{2} e_{1}-b e e_{3}-b f e_{5}, e_{2}, e e_{3}+f e_{5}, e^{2} e_{4}, e^{3} e_{5}, e^{4} e_{6}\right\} .
$$

Then the structure equations become $(0,0,0,12,13,34+25)$. Taking the new dual basis $\left\{e_{2}-e_{3}, e_{1}, e_{2}+e_{3},-e_{4}+e_{5}, e_{4}+e_{5}, 2 e_{6}\right\}$, we find this algebra isomorphic to $\mathfrak{h}_{19+}$. Note that the algebras $\mathfrak{h}_{19+}$ and $\mathfrak{h}_{19-}$ over complex number.

The last cases are due to $a=d=0$. If it is not already equivalent to a previous case, they are equivalent to one of the following:

$$
\begin{gathered}
\mathfrak{h}_{4}: \quad \rho\left(e_{1}\right)=-\left(\begin{array}{lll}
0 & 0 & 0 \\
0 & 0 & 0 \\
0 & 1 & 0
\end{array}\right), \quad \rho\left(e_{3}\right)=-\left(\begin{array}{lll}
0 & 0 & 0 \\
0 & 0 & 0 \\
1 & 0 & 0
\end{array}\right), \quad \rho\left(e_{5}\right)=0, \\
\mathfrak{h}_{8}: \quad \rho\left(e_{1}\right)=0, \quad \rho\left(e_{3}\right)=0, \quad \rho\left(e_{5}\right)=0 .
\end{gathered}
$$

We could exclude $\mathfrak{h}_{19+}$ and $\mathfrak{h}_{19-}$ for further analysis because they do not admit any invariant complex structure [28]. When $\mathfrak{h}_{8}$ is given in (4.19), the integrability of a compatible integrable complex structure as given in (3.8) implies that the algebra $\mathfrak{g}$ is abelian. This contradiction implies that when $\mathfrak{h}_{8}$ admits a semi-direct product structure with a compatible complex structure, then it is the semi-direct product of an abelian subalgebra and an abelian ideal, as given in (4.17).

Except for existence, we obtain an answer to Problem 1.

Theorem 4.1. Suppose that $\mathfrak{h}$ is a six-dimensional nilpotent Lie algebra with an invariant complex structure $J$, given as a totally real semi-direct product $\mathfrak{g} \ltimes_{\rho} V$. Then it is one of the algebras given in the first row of the table below. Its mirror image is a Lagrangian semi-direct product $\mathfrak{h}^{\vee}=\mathfrak{g} \ltimes_{\rho} V^{*}$ given in the corresponding column in the table.

\begin{tabular}{|l||l|l|l|l||l|l|l|l|l|}
\hline$(\mathfrak{h}, J)$ & $\mathfrak{h}_{1}$ & $\mathfrak{h}_{3}$ & $\mathfrak{h}_{8}$ & $\mathfrak{h}_{9}$ & $\mathfrak{h}_{4}$ & $\mathfrak{h}_{6}$ & $\mathfrak{h}_{7}$ & $\mathfrak{h}_{10}$ & $\mathfrak{h}_{11}$ \\
\hline$\left(\mathfrak{h}^{\vee}, \omega_{J}\right)$ & $\mathfrak{h}_{1}$ & $\mathfrak{h}_{6}$ & $\mathfrak{h}_{8}$ & $\mathfrak{h}_{9}$ & $\mathfrak{h}_{7}$ & $\mathfrak{h}_{6}$ & $\mathfrak{h}_{4}$ & $\mathfrak{h}_{10}$ & $\mathfrak{h}_{11}$ \\
\hline
\end{tabular}

In this table, the first four cases are semi-direct products of an abelian ideal with an abelian sub-algebra. The next five cases are semi-direct products of an abelian ideal with the three-dimensional Heisenberg algebra.

We postpone establishing the existence of totally real complex structure $J$ for the semi-direct product algebras in Theorem 4.1 until a proof of Theorem 4.3 is completed. In our proof of Theorem 4.3, we shall only use the "necessity" part of Theorem 4.1. i.e., if a totally real semi-direct structure exists, it has to be one of those given in the table above. 
4.2. Special Lagrangian pairs. Next among the algebras appearing in Theorem 4.1, we study the existence and mirror images of special Lagrangian structures. Since $\mathfrak{h}_{3}$ does not admit invariant symplectic structure, the pair $\left(\mathfrak{h}_{3}, \mathfrak{h}_{6}\right)$ is excluded.

All other cases require further analysis. We begin with the pair $\left(\mathfrak{h}_{6}, \mathfrak{h}_{6}\right)$. The structure equations for $\mathfrak{h}_{6}$ as given in (4.18) are $d e^{4}=e^{12}$ and $d e^{5}=e^{13}$. If the semi-direct product is totally real with respect to an invariant complex structure $J$, then there exists real numbers $a_{i j}$ such that $J e_{2 i-1}=\sum_{j} a_{i j} e_{2 j}$ for $1 \leq i, j \leq 3$. The constraints for $J$ to be integrable are $a_{31}=0, a_{32}=a_{21}$ and $a_{33}=0$. Therefore,

$$
J e_{5}=a_{32} e_{4} \quad \text { or } \quad a_{32} J e_{4}=-e_{5} .
$$

In particular, $a_{21}=a_{32} \neq 0$.

Let $\omega$ be a symplectic structure on $\mathfrak{h}_{6}$ such that $\mathfrak{g}$ and $V$ are both Lagrangian. If $b_{i j}:=\omega\left(e_{i}, e_{j}\right)$, then by using that $\omega$ is closed we obtain that $b_{54}=b_{56}=0$ (recall that $e_{5} \in\left[\mathfrak{h}_{6}, \mathfrak{h}_{6}\right]$ and $e_{4}, e_{6}$ belong to the center) and $b_{52}=b_{43}$. Let us now assume that $\omega$ and $J$ are compatible. Then $\omega\left(J e_{4}, J e_{3}\right)=\omega\left(e_{4}, e_{3}\right)$. It is equivalent to $\omega\left(a_{32} J e_{3}, J e_{3}\right)=a_{32} b_{43}$. By (4.20),

$$
\begin{aligned}
a_{32} b_{43} & =-\omega\left(e_{5}, J e_{3}\right)=-\omega\left(e_{5}, a_{21} e_{2}+a_{22} e_{4}+a_{23} e_{6}\right) \\
& =-a_{21} b_{52}=-a_{21} b_{43}=-a_{32} b_{43} .
\end{aligned}
$$

Since $a_{32} \neq 0$, it is possible only when $b_{43}=0$. As $b_{52}=b_{43}=0$ and $b_{54}=b_{56}=0, \omega$ would have been degenerate. It shows that $h_{6}$ does not admit any special Lagrangian structure with respect to any semi-direct product decomposition.

Since the algebra $\mathfrak{h}_{8}$ is simply the direct sum of a three-dimensional Heisenberg algebra and a trivial algebra, special Lagrangian structures exist in abundance. For example, the structure equation for $\mathfrak{h}_{8}$ is given in (4.17). i.e., $d e^{6}=e^{3} \wedge e^{2}$. Define $J$ and $\omega$ by

$$
J e_{3}=e_{2}, \quad J e_{6}=e_{1}, \quad J e_{4}=e_{5}, \quad \omega=e^{36}+e^{21}+e^{45}
$$

Then the semi-direct product structure of $\mathfrak{h}_{8}$ in (4.17) is special Lagrangian with respect to this pair of $J$ and $\omega$.

Finally, we establish the existence of special Lagrangian structures on the algebras $\mathfrak{h}_{4}, \mathfrak{h}_{7}, \mathfrak{h}_{9}, \mathfrak{h}_{10}$ and $\mathfrak{h}_{11}$. As it turns out, they could be considered as a family of special Lagrangian structures with "jumping" algebraic Lie structures, and hence jumping complex and symplectic structures. 
We fix a basis $\left\{e_{1}, e_{2}, e_{3}, e_{4}, e_{5}, e_{6}\right\}$ of a real vector space $\mathfrak{h}$ and consider also fixed structures $J$ and $\omega$ defined by

$$
J e_{2 j-1}=e_{2 j}, \quad J e_{2 j}=-e_{2 j-1}, \quad \omega=e^{16}-e^{25}+e^{34} .
$$

The 2 -form $\omega$ is type $(1,1)$ with respect to $J$, and the non-degenerate symmetric bilinear form $g(-,-):=\omega(-, J-)$ has signature $(4,2)$. If $\mathfrak{g}=$ $\left\langle e_{1}, e_{3}, e_{5}\right\rangle$ and $V=\left\langle e_{2}, e_{4}, e_{6}\right\rangle$ then $\mathfrak{g}$ and $V$ are totally real with respect to $J$ and maximally isotropic with respect to $\omega$.

Let $(a, b, c, d)$ be real numbers. For each member of the family of Lie brackets

$$
(0,0,0, a 12, b 13, c 14+d 23)
$$

the corresponding Lie algebra $\mathfrak{h}$ is the semi-direct product $\mathfrak{h}=\mathfrak{g} \ltimes V$, and the ideal $V$ is abelian. The constraint on $\mathfrak{h}_{a, b}$ so that $\omega$ is closed is equivalent to $a+b+d=0$. To find the constraints on $\mathfrak{h}_{a, b}$ so that $J$ is integral, we choose a basis for the $(1,0)$-forms with $\omega^{j}=e^{2 j-1}+i e^{2 j}, 1 \leq j \leq 3$. As $d \omega^{1}=0$ and $d \omega^{2}$ is type $(1,1)$, the sole constraint is due to $\omega^{1} \wedge \omega^{2} \wedge d \omega^{3}=0$. Given the structure equations, the integrability of $J$ is equivalent to $b-c-d=0$. It then follows that for any $a, b \in \mathbb{R}$,

$$
\left(\mathfrak{h}_{a, b}=(0,0,0, a 12, b 13,(a+2 b) 14-(a+b) 23), J, \omega\right),
$$

is a family of special Lagrangian pseudo-Kähler structures on nilpotent Lie algebras. Since a non-zero scalar multiple of a Lie bracket gives rise to just a homothetic change in the metric, we will restrict ourselves to the curve $\left\{\mathfrak{h}_{a, b}: a^{2}+b^{2}=1, b \geq 0\right\}$. The following isomorphisms can be checked by using the new basis on the right:

$$
\begin{aligned}
& \mathfrak{h}_{ \pm 1,0} \simeq \mathfrak{h}_{9}, \quad\left\{e_{2}, e_{1}, e_{5}, e_{3},-e_{4},-e_{6}\right\} \\
& \mathfrak{h}_{0,1} \simeq \mathfrak{h}_{4}, \quad\left\{e_{1}, e_{3}, e_{2}, \frac{1}{2} e_{4}, e_{5}, e_{6}\right\}
\end{aligned}
$$

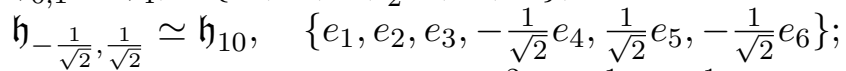

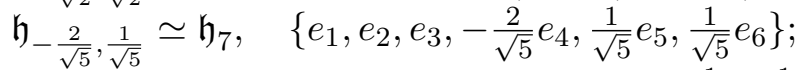

$$
\begin{aligned}
& \mathfrak{h}_{a, b} \simeq \mathfrak{h}_{11} \text { if } a, b, a+2 b, a+b \neq 0, \quad\left\{r e_{1}, \frac{1}{r} e_{2}, \frac{1}{r} e_{3}, a e_{4}, b e_{5}, r a(a+2 b) e_{6}\right\},
\end{aligned}
$$

where $r=-\left(\frac{a+b}{a(a+2 b)}\right)^{1 / 3}$.

Isomorphism classes of such structures translate themselves in this context as the orbits of the natural action of the group

$$
\mathrm{U}(2,1)=\left\{\varphi \in \mathrm{GL}_{6}(\mathbb{R}): \varphi J \varphi^{-1}=J, \omega(\varphi \cdot, \varphi \cdot)=\omega\right\}
$$


on the space of Lie brackets $[\mathbf{2 4}]$. Let $\mathfrak{h}_{a, b}, \mathfrak{h}_{a^{\prime}, b^{\prime}}$ be two points in the curve isomorphic as Lie algebras to $\mathfrak{h}_{11}$, and assume there exists $\varphi \in \mathrm{U}(2,1)$ such that

$$
\varphi \cdot[\cdot, \cdot]_{a, b}:=\varphi\left[\varphi^{-1} \cdot, \varphi^{-1} \cdot\right]_{a, b}=[\cdot, \cdot]_{a^{\prime}, b^{\prime}} \cdot
$$

By using that $\varphi$ must leave invariant the center $\mathfrak{z}=\left\langle e_{5}, e_{6}\right\rangle_{\mathbb{R}}$, the derived subalgebra $[\mathfrak{h}, \mathfrak{h}]=\left\langle e_{4}, e_{5}, e_{6}\right\rangle_{\mathbb{R}}$, the subalgebras $[\mathfrak{h},[\mathfrak{h}, \mathfrak{h}]]=\left\langle e_{6}\right\rangle_{\mathbb{R}}$ and $\{x \in$ $\mathfrak{h}:[x, \mathfrak{h}] \subset \mathfrak{z}\}=\left\langle e_{3}, e_{4}\right\rangle_{\mathbb{R}}$, as well as their orthogonal complements relative to both $\omega$ and the metric $g=\omega(\cdot, J \cdot)$, one can easily show that the matrix of $\varphi$ with respect to the basis $\left\{e_{1}, e_{2}, \ldots, e_{6}\right\}$ is necessarily diagonal, and so it is a diagonal matrix with entries $s, s, \pm 1, \pm 1,1 / s, 1 / s$ with $s \neq 0$. It follows that $a^{\prime}= \pm \frac{1}{s^{2}} a, b^{\prime}= \pm \frac{1}{s^{2}} b$ and hence $\left(a^{\prime}, b^{\prime}\right)= \pm(a, b)$.

We summarize the results obtained above in the following proposition.

Proposition 4.2. With complex structure $J$ and symplectic structure $\omega$ given in (4.21), with $t \in[0, \pi]$ the function

$$
((0,0,0,(\cos t) 12,(\sin t) 13,(\cos t+2 \sin t) 14-(\cos t+\sin t) 23), J, \omega)
$$

determines a closed curve in the moduli space of isomorphism classes of special Lagrangian pseudo-Kähler structures on the space of six-dimensional nilpotent Lie algebras. It contains exactly one structure on each one of $\mathfrak{h}_{4}$ $(t=\pi / 2), \mathfrak{h}_{7}\left(t=\arctan \left(-\frac{1}{2}\right)\right), \mathfrak{h}_{9}(t=0, \pi), \mathfrak{h}_{10}(t=3 \pi / 4)$, and the remaining is a continuous family on $\mathfrak{h}_{11}$.

As a consequence of Theorem 4.1, the non-existence on special Lagrangian semi-direct product structures on $\mathfrak{h}_{3}$ and $\mathfrak{h}_{6}$, and the existence for the other algebras, we obtain a complete answer to Problem 2.

Theorem 4.3. Suppose that a six-dimensional nilpotent algebra admits a special Lagrangian semi-direct product structure, then the algebra is isomorphic to one of the following: $\mathfrak{h}_{1}, \mathfrak{h}_{4}, \mathfrak{h}_{7}, \mathfrak{h}_{8}, \mathfrak{h}_{9}, \mathfrak{h}_{10}, \mathfrak{h}_{11}$. Their weak mirror images are, respectively, given below:

\begin{tabular}{|l||l|l|l|l|l|l|l|}
\hline$(\mathfrak{h}, J, \omega)$ & $\mathfrak{h}_{1}$ & $\mathfrak{h}_{4}$ & $\mathfrak{h}_{7}$ & $\mathfrak{h}_{8}$ & $\mathfrak{h}_{9}$ & $\mathfrak{h}_{10}$ & $\mathfrak{h}_{11}$ \\
\hline$\left(\mathfrak{h}^{\vee}, \omega_{J}, J_{\omega}\right)$ & $\mathfrak{h}_{1}$ & $\mathfrak{h}_{7}$ & $\mathfrak{h}_{4}$ & $\mathfrak{h}_{8}$ & $\mathfrak{h}_{9}$ & $\mathfrak{h}_{10}$ & $\mathfrak{h}_{11}$ \\
\hline
\end{tabular}

In addition,

- All special Lagrangian semi-direct product structure $(J, \omega)$ on $\mathfrak{h}_{1}, \mathfrak{h}_{8}$, $\mathfrak{h}_{9}$ and $\mathfrak{h}_{10}$ are self-mirror. It means that there is an isomorphism $\mathfrak{h}_{\ell} \cong \mathfrak{h}_{\ell}^{\vee}$ and there are quasi-isomorphisms.

$$
\operatorname{DGA}\left(\mathfrak{h}_{\ell}^{\vee}, \omega_{J}\right) \approx \operatorname{DGA}\left(\mathfrak{h}_{\ell}, \omega\right) \approx \operatorname{DGA}\left(\mathfrak{h}_{\ell}^{\vee}, J_{\omega}\right) \approx \operatorname{DGA}\left(\mathfrak{h}_{\ell}, J\right) .
$$

- The algebras $\mathfrak{h}_{7}$ and $\mathfrak{h}_{4}$ form a mirror pair of special Lagrangian semidirect products. 
- The mirror of a special Lagrangian semi-direct structure on $\mathfrak{h}_{11}$ is again a special Lagrangian semi-direct structure on $\mathfrak{h}_{11}$, but the two pseudo-Kähler structures are not equivalent.

Proof. The isomorphisms between DGA $(\mathfrak{h}, J)$ and $\operatorname{DGA}\left(\mathfrak{h}^{\vee}, \omega_{J}\right)$ are given by Theorem 3.10 and the identification of algebraic mirrors $\mathfrak{h}^{\vee}$ as given in Theorem 4.1. The validity of the claim on $\mathfrak{h}_{11}$ is due to an analysis in $[\mathbf{1 4}$, Section 5.5].

4.3. Completing a proof for Theorem 4.1. We need to establish the existence of totally real semi-direct product structures on each algebra in the table of Theorem 4.1. Except for $\mathfrak{h}_{3}$ and $\mathfrak{h}_{6}$, in the last paragraph we have established the existence of special Lagrangian structures, including a totally real complex structure.

Our analysis on $\mathfrak{h}_{6}$ in Section 4.2 has already indicated the constrains on a totally real complex structure on the semi-direct product given in (4.18). For instance, one could choose

$$
J e_{1}=e_{2}, \quad J e_{3}=e_{6}, \quad J e_{5}=e_{4} .
$$

The semi-direct product structure on the algebra $\mathfrak{h}_{3}$ is given in (4.16). Its structure question is $d e^{6}=e^{14}+e^{32}$. Up to equivalence, this algebra has a unique invariant complex structure [28, Proposition 3.4]. In our basis, it is

$$
J e_{1}=e_{4}, \quad J e_{3}=e_{2}, \quad J e_{5}=e_{6} .
$$

It is totally real with respect to the semi-direct structure in (4.16).

Thus, we establish the existence of totally real semi-direct structures on each algebra given in the table of Theorem 4.1.

\section{References}

[1] A. Andrada and S. Salamon, Complex product structures on Lie algebras, Forum Math. 17 (2005), 261-295.

[2] D. Arinkin and A. Polishchuk, Fukaya category and Fourier transform, in 'Winter School on Mirror Symmetry, Vector Bundles and Lagrangian Submanifolds', (C. Vafa \& S. T. Yau eds.), Studies in Advanced Mathematics, 23, AMS/International Press, Providence, 2001, $261-274$.

[3] C. Benson and C. S. Gordon, Kähler and symplectic structures on nilmanifolds, Topology 27 (1988), 513-518.

[4] C. Benson and C. S. Gordon, Kähler structures on compact solvmanifolds, Proc. Amer. Math. Soc. 108 (1990), 971-980.

[5] M. L. Barberis and I. G. Dotti, Complex structures on affine motion groups, Q. J. Math. 55 (2004), 375-389.

[6] O. Baues, Left-symmetric algebras for $\mathfrak{g l}(n)$, Trans. Amer. Math. Soc. 351 (1999), 2979-2996.

[7] O. Baues and V. Cortés, Aspherical Kähler manifolds with solvable fundamental group, Geometriae Dedicata 122 (1) (2006) (arXiv:math.DG/0601616). 
[8] O. Ben-Bassat, Mirror symmetry and generalized complex manifolds. I 8 II, The transform on vector bundles, spinors, and branes, J. Geom. Phys. 56 (2006), 533558, 1096-1115.

[9] Y. Benoist, Une nilvariété non affine, J. Differential Geom. 41 (1995), 21-52.

[10] M. Nguiffo Boyom, Variétés symplectiques affines, Manuscripta Math. 64 (1989), $1-33$.

[11] D. Burde, Affine structures on nilmanifolds, Internat. J. Math. 7 (1996), 599-616.

[12] D. Burde, Simple left-symmetric algebras with solvable Lie algebra, Manuscripta Math. 95 (1998), 397-411.

[13] B. Y. Chu, Symplectic homogeneous spaces, Trans. Amer. Math. Soc. 197 (1974), 145-159.

[14] R. Cleyton and Y. S. Poon, Differential Gerstenhaber algebras associated to nilpotent algebras, Asian J. Math. 12 (2008), 225-250.

[15] S. Console and A. Fino, Dolbeault cohomology of compact nilmanifolds, Transform. Groups 6 (2001), 111-124.

[16] L. A. Cordero, M. Fernández, A. Gray and L. Ugarte, Nilpotent complex structures on compact nilmanifolds, Rend. Circolo Mat. Palermo 49 (suppl) (1997), 83-100.

[17] L. A. Cordero, M. Fernández, A. Gray and L. Ugarte, Compact nilmanifolds with nilpotent complex structures: Dolbeault cohomology, Trans. Amer. Math. Soc. 352 (2000), 5405-5433.

[18] M. Goze and Y. Khakimdjanov, Nilpotent Lie algebras, Kluwer Academic Publishers, Dordrecht, 1996.

[19] M. Gross and B. Siebert, From real affine geometry to complex geometry, preprint (arXiv.org:math/0703822).

[20] K. Hasegawa, Minimal models of nilmanifolds, Proc. Amer. Math. Soc. 106 (1989), 65-71.

[21] K. Hasegawa, A note on compact solvmanifolds with Kähler structures, Osaka J. Math. 43 (2006), 131-135.

[22] N. J. Hitchin, Lectures on special Lagrangian submanifolds, in 'Winter School on Mirror Symmetry, Vector Bundles and Lagrangian Submanifolds' (C. Vafa, and S. T. Yau eds.), Studies in Advanced Mathematics 23, AMS/International Press, Providence 2001, 151-182, (arXiv:math.DG/9907034).

[23] W. M. Goldman and J. J. Millson, The homotopy invariance of the Kuranishi space, Illinois J. Math. 34 (1990), 397-368.

[24] J. Lauret, A canonical compatible metric for geometric structures on nilmanifolds, Ann. Global Analy. Geom. 30 (2006), 107-138 (arXiv: math.DG/ 0410579).

[25] S. A. Merkulov, A note on extended complex manifolds. Symplectic and contact topology: interactions and perspectives (Toronto, ON/Montreal, QC, 2001), 145-155, Fields Inst. Commun., 35, Amer. Math. Soc., Providence, RI, 2003.

[26] Y. S. Poon, Extended deformation of Kodaira surfaces, J. Reine Angew. Math. 590 (2006), 45-65.

[27] S. Rollenske, Nilmanifolds: complex structure geometry and deformations, preprint (arXiv.org:math/0709.9467).

[28] S. Salamon, Complex structures on nilpotent Lie algebras, J. Pure Appl. Algebra 157 (2001), 311-333. 
[29] A. Strominger, S. T. Yau and E. Zaslow, Mirror symmetry in T-duality, Nucl. Phys. B 479 (1996), 243-259.

[30] J. Zhou, Homological perturbation theory and mirror symmetry, Acta Math. Sinica, English Version. 19 (2003), 695-714.

INSTITUTE FÜR MATHEMATIK

Humboldt UNIVERSITÄT ZU BERLIN

UNTER DEN LINDEN 6

10099 BERLIN

GERMANY

E-mail address: cleyton@mathematik.hu-berlin.de

FAMAF AND CIEM

Universidad Nacional de Córdoba

5000 Córdoba, Argentina

E-mail address: lauret@mate.uncor.edu

Department of Mathematics

University OF CALIFORNIA, Riverside

CA 92521

USA

E-mail address: ypoon@ucr.edu

Received 8/08/2008, accepted 04/01/2009

Y.S.P. would like to thank Conan Leung for stimulating conversations. R.C. was supported by the Volkswagen Foundation and the SFB 647 of the DFG. R.C. thank UC Riverside for support during his visit in 2008. Thanks are also due to J. Heber and I. Dotti for pointing authors to some references they might otherwise have missed. 
